\title{
BMJ Open Effectiveness of a new one-hour blood pressure monitoring method to diagnose hypertension: a diagnostic accuracy clinical trial protocol
}

\author{
Luis González-de Paz, ${ }^{\oplus 1,2}$ Belchin Kostov, ${ }^{2,3}$ Maria del Carme Alvira-Balada, ${ }^{2,4}$ \\ Cristina Colungo, ${ }^{2,4}$ Noemí García, ${ }^{4}$ Silvia Roura, ${ }^{5}$ Esther Blat, ${ }^{1}$ \\ Cristina Sierra-Benito, ${ }^{6}$ Josep Miquel Sotoca-Momblona, ${ }^{7}$ Jaume Benavent-Areu, ${ }^{8}$ \\ Eva Sánchez, ${ }^{4}$ Antoni Sisó-Almirall, ${ }^{1,2}$ Eva Sanchez on behalf of the OMBP Group
}

To cite: González-de Paz L, Kostov B, Alvira-Balada MdC et al. Effectiveness of a new one-hour blood pressure monitoring method to diagnose hypertension: a diagnostic accuracy clinical trial protocol. BMJ Open 2019;9:e029268. doi:10.1136/ bmjopen-2019-029268

- Pre-publication history and additional material is published online only. To view please visit the journal online (http://dx.doi. org/10.1136/bmjopen-2019029268).

Received 22 January 2019

Revised 29 April 2019

Accepted 2 May 2019
D) Check for updates

(c) Author(s) (or their employer(s)) 2019. Re-use permitted under CC BY-NC. No commercial re-use. See rights and permissions. Published by BMJ.

For numbered affiliations see end of article.

\section{Correspondence to}

Dr Luis González-de Paz; gonzalezdepaz@hotmail.com, Igonzale@clinic.cat

\section{ABSTRACT}

Introduction 24-hour ambulatory blood pressure monitoring (ABPM) is the gold standard diagnostic method for hypertension, but has some shortcomings in clinical practice while clinical settings often lack sufficient devices to accommodate all patients with suspected hypertension. Home blood pressure monitoring (HBPM) and office blood pressure monitoring (OBPM) also have shortcomings, such as the white coat effect or a lack of accuracy. This study aims to study the validity of a new method of diagnosing hypertension consisting of monitoring blood pressure (BP) for 1 hour and comparing it with OBPM and HBPM and examining the sensitivity and specificity of this method compared with 24-hour ABPM. The patient experience will be examined in each method.

Methods and analysis A minimum sample of 214 patients requiring a diagnostic test for hypertension from three urban primary healthcare centres will be included. Participants will undergo 24-hour ABPM, 1-hour BP measurement (1-BPM), OBPM for three consecutive weeks and HBPM. Patients will follow a random sequence to first receive 24-hour ABPM or 1-hour ABPM. Daytime 24-hour ABPM records will be compared with the other monitoring methods using the correlation coefficient and Bland Altman plots. The kappa concordance index and the sensitivity and specificity of the methods will be calculated. The patient's experience will be studied, with selected indicators of efficiency and satisfaction calculated using parametric tests.

Ethics and dissemination The protocol has been authorised by the research ethics committee of the Hospital Clinic of Barcelona (Ref. HCB/2014/0615): protocol details and amendments will be recorded and reported to ClinicalTrials.com. The results will be disseminated in peer-reviewed literature, and to policy makers and healthcare partners.

Trial registration NCT03147573; Pre-results.

\section{BACKGROUND}

Hypertension, a leading cardiovascular risk factor that causes premature morbidity and mortality, is associated with an increased risk of cerebrovascular disease (haemorrhagic

\section{Strengths and limitations}

- This trial will examine whether the sensitivity and specificity of 1-hour blood pressure (BP) monitoring is comparable with 24 hours monitoring and other conventional methods of diagnosing hypertension.

- Validation of the new method would not allow nighttime recording of $\mathrm{BP}$ and does not seek to substitute 24 hours monitoring.

- This study will examine the patient experience of the new BP monitoring methods studied.

or ischaemic), coronary heart disease, heart failure, chronic renal failure, peripheral vascular disease, cognitive impairment and premature death. ${ }^{2}$ In high-income countries, the age-standardised prevalence of hypertension rises to $28.5 \%$ (95\% CI $27.3 \%$ to $29.7 \%)^{3}$

Current evidence suggests that patients with systolic blood pressure $(\mathrm{SBP}) \geq 140 \mathrm{~mm}$ $\mathrm{Hg}$ and/or diastolic blood pressure (DBP) $\geq 90 \mathrm{~mm} \mathrm{Hg}$ require treatment to reduce the risk of cardiovascular and kidney disease. ${ }^{4}$ However, not all patients with these values have a high cardiovascular risk, sometimes due to factors such as white coat hypertension. The diagnosis of hypertension requires accurate blood pressure (BP) monitoring to determine whether the patient has constant high $\mathrm{BP}$ values and this requires various determinations. At present, clinical guidelines accept three methods of BP monitoring: 24-hour ambulatory BP monitoring (24-hour ABPM), home BP monitoring (HBPM) and office BP monitoring (OBPM). ${ }^{4-6}$

24-hour ABPM, the gold standard, consists of applying an automatic oscillometer sphygmomanometer connected to a cuff on the patient's arm. The device records BP values 
for 20 or 30 min during 24 hours. ${ }^{4}$ This identifies differences between day and night and the risk associated with hypertension. ${ }^{78}$ However, the high incidence of hypertension means it cannot be carried out in all patients who require it, and 24-hour ABPM is not always available for all primary healthcare patients and is uncomfortable. ${ }^{9}$

HBPM measures BP at home or in the pharmacy. Determinations may be made by patients or relatives after receiving instruction. ${ }^{10}$ This method avoids bias due to the white coat effect. However, the benefits may be lost due to incorrect application of the technique or a lack of standardisation of the measuring equipment (measurement with a mercury sphygmomanometer is still used). ${ }^{11}$

OBPM consists of taking the BP measures at the clinic for several days. After determining the reference arm, three determinations are made once a week for three consecutive weeks and the mean of these determinations is calculated. The disadvantages of OBPM are the overdiagnosis of hypertension, unnecessary treatments in $15 \%-30 \%$ of patients, ${ }^{8}$ the inability to detect sudden changes in BP, measurement errors, the limited number of records that can be made, and the risk of confusing isolated hypertension, the white coat effect and the lack of correlation with real BP. ${ }^{12}{ }^{13}$ Other drawbacks are the lack of data on the prognostic value and the definition. ${ }^{71415}$ However, despite its disadvantages, clinicians often perceive that OBPM is controllable and verifiable, and therefore it persists as the preferred method, including in large population studies and pharmacological clinical trials with a required diagnosis of hypertension. ${ }^{12} 16$

Recently, two studies have examined the effectiveness of two new methods which use a modified 24-hour ABPM device. One takes a set of measurements during $30 \mathrm{~min}$ and the other during $60 \mathrm{~min} .{ }^{17}{ }^{18}$ These methods have some of the advantages of OBPM: the clinician can verify and control the process because the patient is in the clinic but avoiding the frequent white coat effect. In 1 hour-BP measurement (1-BPM), mean daytime BP values were similar to 24-hour ABPM, with a sensitivity of $85.2 \%$ (95\% CI $67.5 \%$ to $94.1 \%$ ) and a specificity of $92 \%$ (95\% CI $83.6 \%$ to $96.3 \%$ ) for masked white coat hypertension. ${ }^{18}$ However, 1-BPM requires further study for use in clinical practice, and the patient experience in terms of handling, satisfaction, understanding and adherence is unclear. Patient involvement in the evaluation is required to improve the quality of care, and to increase effectiveness and the patient's sensitivity to their health status according to their perceived needs. ${ }^{19}$ However, this has not been widely reported in previous studies and current BP monitoring methods have not been studied in the light of patient experience. ${ }^{13}$

The European, North American and British guidelines recommend the diagnosis should be made after verifying $\mathrm{BP}$ values with an ambulatory method involving various measurements or, at best, using 24-hour ABPM. ${ }^{4-6}$ However, all methods of measuring hypertension in daily clinical practice have shown important shortcomings related to the accuracy of monitoring, and 24-hour ABPM is not feasible in all cases. ${ }^{9}$ The aim of this study is to examine the accuracy of the 1-BPM method against the out-of-office and office methods, taking 24-hour ABPM as the gold standard.

\section{METHODS/DESIGN}

\section{Study objectives}

To study the validity (sensitivity and specificity) of 1-BPM versus OBPM in three visits and HBPM, using 24-hour ABPM as the reference during the daytime period, and to examine patient acceptance and experience of the methods and compare the cost of 1-BPM with the other methods.

\section{Design}

This study protocol presents a diagnostic accuracy study of BP measurement using ABPM. Patients requiring a diagnostic test for high BP will be prospectively included. All participants will undergo 24-hour ABPM. The specificity and sensibility will be studied with respect to 1-BPM, HBPM and OBPM at three visits. The trial registration identifier in ClinicalTrials.gov web is CT03147573, registered 10 May 2017.

\section{Study population}

Patients assigned to a primary healthcare centre (PHC) referred for a diagnostic test for hypertension by the family physician.

\section{Inclusion and exclusion criteria}

Inclusion criteria will be as follows: (1) age $\geq 18$ years, (2) patients seen routinely in the study PHCs, (3) voluntary participation and (4) family physician referral for diagnosis of high BP. Exclusion criteria will be as follows: (1) severe physical or cognitive limitations, (2) previous episodes of arrhythmia, atrial fibrillation with rapid ventricular response, frequent ventricular extra-systole or other arrhythmias, (3) Parkinson's disease or any other condition causing permanent tremor, (4) arm circumference $>42 \mathrm{~cm}$, (5) arterial-venous fistula in the arm, (6) mental disorders or intolerance to BP measurement method, (7) inability to attend the study PHC and (8) programmed hospitalisation during the study period.

\section{Clinical setting}

Participants will be recruited from three urban PHCs located in Barcelona (Spain), which include 54 family physicians and 56 community nurses who serve 95185 patients. In 2017, $65.21 \%$ of patients were seen $\geq 1$ time. Participant inclusion commenced in June 2017, and the estimated completion date is June 2019.

\section{Outcome measurements}

\section{Primary outcome}

Diagnosis of hypertension, according to The European Society of Cardiology and The European Society of Hypertension (ESC/ESH) Arterial Hypertension guidelines definitions ${ }^{4}$ (excluding 1-BPM): 
- 24-hour ABPM: daytime mean of $\geq 135 \mathrm{~mm} \mathrm{Hg} \mathrm{SBP}$ and/or $\geq 85 \mathrm{~mm} \mathrm{Hg}$ DBP.

- OBPM: mean of three visits $\geq 140 \mathrm{~mm} \mathrm{Hg}$ and/or DBP) $\geq 90 \mathrm{~mm} \mathrm{Hg}$.

- HBPM: mean of $\geq 135 \mathrm{~mm} \mathrm{Hg} \mathrm{SBP}$ and/or $\geq 85 \mathrm{~mm} \mathrm{Hg}$ DBP.

- 1-BPM: mean of $\geq 135 \mathrm{~mm} \mathrm{Hg} \mathrm{SBP}$ and/or $\geq 85 \mathrm{~mm} \mathrm{Hg}$ DBP.

\section{Patient experience}

Satisfaction with the time necessary to obtain the test result, time required by the patient to obtain the result (including time of travel to the centre), comfort of the test and opinion about recommending it to another patient. These variables will be collected using visual analogic scales (online supplementary file 1); nurse collaborators will assist participants if required.

\section{Cost}

Cost of the visit, number of visits needed to carry out the diagnostic procedure, any spending reported by the participant, cost of travel to the health centre (if applicable) and other reported expenses.

\section{Other variables}

Sociodemographic: age and sex, educational level, social class; clinical variables: weight, height and body mass index, abdominal perimeter, ECG, cardiovascular risk factors, smoking, alcohol consumption, level of physical activity, diabetes mellitus, dyslipidaemia, obesity, previous prescription of antihypertensive medication and type.

\section{BP measurement methods}

\section{4-hour ABPM}

BP measurement will be made using Oscar 2, model 250, 24-hour ABPM, Suntech Medical, which is approved by the US Food and Drug Administration..$^{20}$ Using a standard cuff adapted to the arm, the device is activated at intervals for 24 hours: every $20 \mathrm{~min}$ during the daytime and every 30 min during night-time. A minimum of $70 \%$ of $\mathrm{BP}$ records are needed for a valid ABPM measurement. The device stores the BP records in an internal memory that is downloaded once the test finishes the next day. The characteristics are shown elsewhere. ${ }^{21}$

\section{1-BPM}

Measurement of BP for 1 hour using the 24-hour ABPM device. $\mathrm{BP}$ will be measured every $5 \mathrm{~min}$. During the hour, the patient will remain in a quiet room in the primary healthcare centre, without walking actively, eating or smoking. After 1 hour, the device and arm cuff will be removed.

\section{OBPM}

A standard device will be used (Omron m6 AC, Japan). The patient must not have smoked tobacco, drunk coffee or other caffeine-containing drinks in the previous $30 \mathrm{~min}$ or had a large meal in the previous 2 hours, complain of pain or anxiety and must not have psychomotor agitation.
The patient will be seated with their arms crossed, the legs uncrossed and the feet touching the floor. DBP and SBP will be determined in the two arms, and the arm with the highest BP will be selected; if both are equal either will be chosen. After 5 min, a new determination will be made, which will be the one recorded in the data collection notebook. In following visits, BP will be determined following the same procedure but only in the reference arm.

\section{HBPM}

HBPM will be carried out according to daily clinical practice. All participants will be asked to provide at least three BP measurements over 3 days. Nurses will check whether the participant has an adequate BP monitor (a semiautomatic, validated BP monitor); if they do not have access to a validated BP device at home, they will be asked to measure the BP in the pharmacy. Participants will receive instructions and an information sheet on the BP measuring method. BP should be measured in a quiet room without noise or interruptions. Patients should be relaxed and should not drink, eat, smoke or exercise during the previous half hour. Patients should not talk during the measurement and should rest for $5 \mathrm{~min}$ beforehand. They should sit comfortably with the back resting on the back of the chair, with the legs uncrossed and arms not constrained by clothing. The cuff should be placed on the arm, 2 or $3 \mathrm{~cm}$ above the elbow, leaving the hand on the back and the elbow slightly flexed at the height of the heart. Two measurements will be made at least 2 min apart and the mean of the two will be used. Measurement in the pharmacy will follow the recommendations of pharmacy employees, as in usual practice. Table 1 summarises the number of days/hours/visits needed, measurements obtained and the threshold used to diagnose hypertension in each of the four methods.

\section{Study procedures}

The study will have a total of three visits. Figure 1 presents an overview of the tasks carried out at each study visit and a flowchart of the process. The tasks are shown below.

\section{Visit 0}

Patients with inclusion criteria seen by PHC physicians or nurses will be invited to participate. If the potential participant shows interest, they will receive information on the study requirements. Any patient doubts will be answered and clinicians will offer a brochure with detailed information on the study. At this visit, the nurse will measure the $\mathrm{BP}$ and determine the reference arm, will measure the first OBPM and will request a HBPM. The next day, the nurse will call the patient to confirm inclusion and arrange the next visit.

\section{Visit 1}

Between 5 and 7 days after visit 0 , the participant will attend visit 1 with the nurse. All nurses will be instructed by the study coordinator in data collection and the study procedures. Before starting the procedures, they will 
Table 1 Summary of the methods according the time required, the number of measurements of BP and the threshold of BP to diagnose hypertension

\begin{tabular}{llll}
\hline Method & $\begin{array}{l}\text { Number of days/hours/visits } \\
\text { needed }\end{array}$ & $\begin{array}{l}\text { Number measurements } \\
\text { obtained }\end{array}$ & Hypertension threshold \\
\hline $\begin{array}{l}\text { 24-hour ABPM: } \\
\text { daytime }\end{array}$ & 24 hours & 49 to 52 & $\geq 135 \mathrm{~mm} \mathrm{Hg} \mathrm{SBP} \mathrm{and/or} \geq 85 \mathrm{~mm} \mathrm{Hg} \mathrm{DBP}$ \\
OBPM & $\begin{array}{l}\text { Three consultations: once a week } \\
\text { in three consecutive weeks }\end{array}$ & 3 & $\geq 140 \mathrm{~mm} \mathrm{Hg} \mathrm{SBP} \mathrm{and/or} \geq 90 \mathrm{~mm} \mathrm{Hg} \mathrm{DBP}$ \\
HBPM & $\begin{array}{l}\text { Two consultations } \\
\text { 1-BPM }\end{array}$ & 3 & $\geq 135 \mathrm{~mm} \mathrm{Hg} \mathrm{SBP} \mathrm{and/or} \geq 85 \mathrm{~mm} \mathrm{Hg} \mathrm{DBP}$ \\
\hline
\end{tabular}

1-BPM, 1-hour blood pressure monitoring; ABPM, ambulatory blood pressure monitoring; BP, blood pressure; DBP, diastolic BP; HBPM, home BP monitoring; OBPM, office BP monitoring; SBP, systolic BP.

review the study aims and the patient will be asked to sign an informed consent. Thereafter, the nurse will record anthropometric variables and carry out the second determination of OBPM and patients will follow the 1-BPM or 24-hour ABPM according a randomisation sequence. If 24-hour ABPM is chosen, a visit will be arranged from Monday to Thursday (not Friday because the device must be removed the next day at the same time).

At the end of this visit, an electrocardiographic record will be made. A new HBPM will be requested.

\section{Visit 2}

Between 5 and 7 days after visit 1, the third OBPM determination will be made. 1-BPM will be administered to patients who underwent 24-hour ABPM during visit 2 and 1-BPM in patients in whom 24-hour ABPM was carried out during visit 1 . The nurse will collect data on the variables concerning the patient's experience of the four methods using the patient experience sheet (online supplementary appendix 1). The nurse will record the HBPM participants have provided. After completion, the patient will be referred to their general practitioner to assess the results.

\section{Participant discontinuation}

Participants who start any drug treatment to treat hypertension before visit 2, or do not attend any of the visits for three consecutive weeks will be withdrawn from the study. To promote retention, the nurse will phone participants before the visit and will change the date of the visit to another day of the same week if the participant requests.

\section{1-BPM and 24-hour ABPM sequence randomisation}

As 1-BPM and 24-hour ABPM use the same device, there might be some adaptation effect, which will be mitigated by randomisation: at visit 1 and visit 2, each participant will be allocated randomly to 1-BPM or 24-hour ABPM.

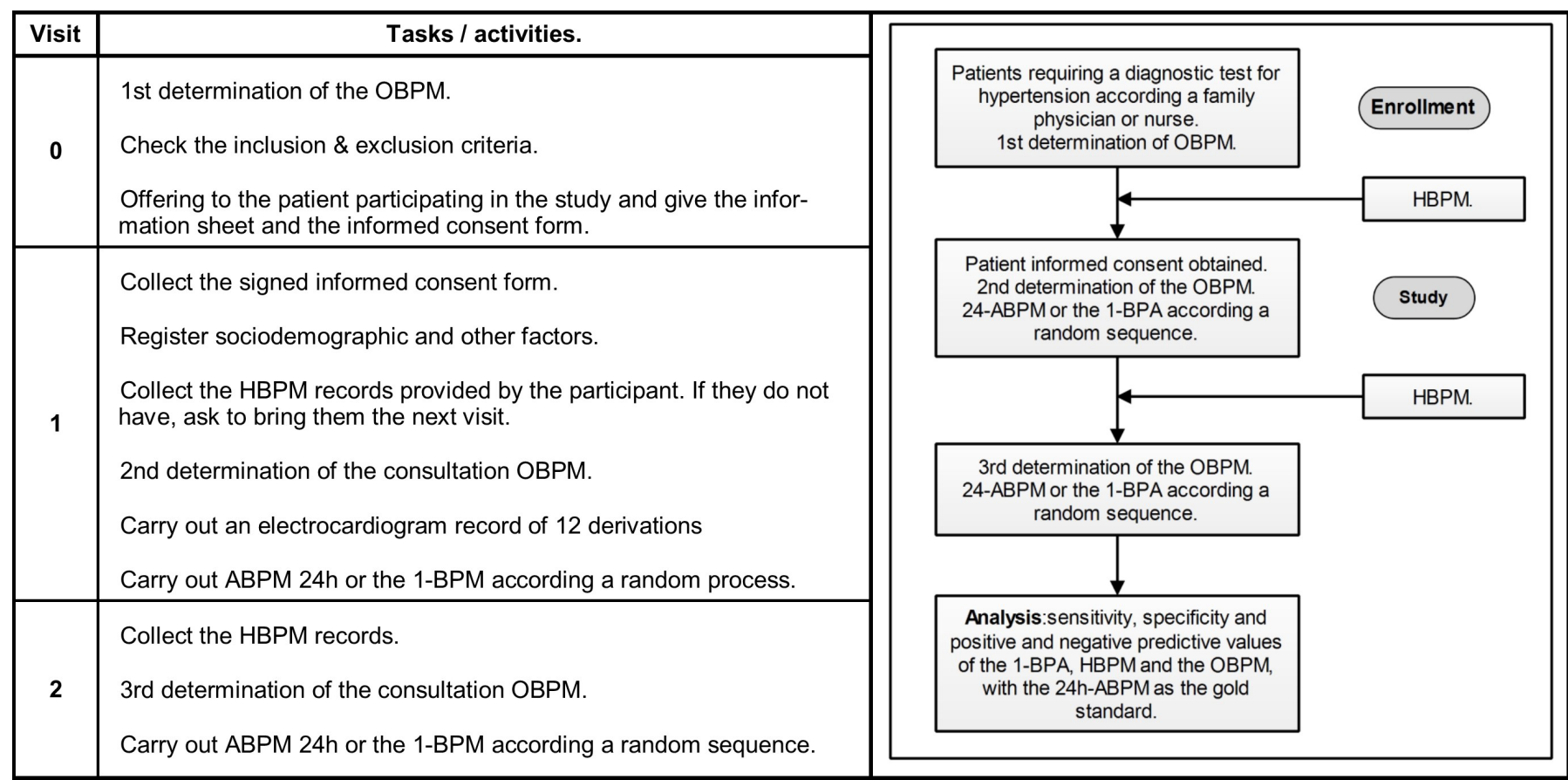

Figure 1 Research visits and content of study assessments (left) and flow chart of study design (right). 1-BPM, 1-hour ambulatory blood pressure monitoring; 24-hour ABPM, 24-hour ambulatory blood pressure monitoring; HBPM, home blood pressure monitoring; OBPM, office blood pressure monitoring. 
The randomisation sequence will be generated previously and will not be changed. We do not foresee that any other randomisation method will be needed to examine the study outcomes.

\section{Statistical analysis}

Categorical variables will be presented as absolute frequency and percentages and continuous variables as mean and SD, or median and IQR. The Shapiro-Wilk normality test will be used to check the normal distribution of variables. Pearson's correlation coefficient will be calculated for 1-BPM, HBPM and OBPM. The Bland-Altman method will be applied with the graphic representation of the correlations of the intervals (the differences between the measurements against the mean) to confirm independence between the differences obtained with each method and the magnitude. The prevalence of white coat hypertension and masked hypertension will be calculated. The proportion of well-classified participants with 1-BPM will be estimated with respect to 24-hour ABPM and HBPM and OBPM, and the kappa index will be calculated to measure the degree of agreement between the four methods, to classify the participants in subpopulations of hypertensive patients. Sensitivity, specificity and positive and negative predictive values will be calculated for the diagnosis of hypertension subtypes. All concordance and correlation results will be based on the means of SBP and DBP readings. To analyse the cost, the mean of the sum of all the cost variables will be analysed and each variable will be analysed separately by calculating the differences between means by analysis of variance. Likewise, the time necessary to obtain the result of the test and the validity with respect to 24-hour ABPM will be analysed. Values of $p<0.05$ will be considered statistically significant. The statistical analysis will be made using the R V.3 for Windows statistical program.

\section{Sample size calculation}

A recent study comparing 1-BPM with 24-hour ABPM found that 1-BPM classified $87.3 \%$ of patients correctly. To achieve a precision of $5 \%$ in the estimate of a proportion with $95 \%$ CIs, assuming the proportion is $87.30 \%$, and taking into account an expected dropout rate of $20 \%$, a minimum of 214 patients will be required.

\section{Patient involvement}

There was no patient or public involvement in the study design. The study aims to analyse regarding the time required by the patient to obtain the result, including the time of travel to the centre, the comfort of the test and opinions on recommending it to another patient.

\section{Ethics and dissemination}

The protocol presents a health technology trial to study a new test with a medical device, but is not a medicine. The procedures will follow Spanish and Catalan laws. Researchers will follow the ethical standards of the Declaration of Helsinki for biomedical studies and the activities described will follow the Code of Good Practice in
Clinical Research. The protocol has been authorised by the research ethics committee of the Hospital Clinic of Barcelona (Ref. HCB/2014/0615). Authorisation will be sought from the research ethics committee for protocol modifications, which will be reported to Clinical Trials. gov. The results will be presented in the scientific literature, and to healthcare partners.

\section{Confidentiality and data management}

All study-related information will be stored securely at the study sites. All participant information will be stored in locked file cabinets in areas with limited access. Information from participants will be identified by a coded identification number only to maintain participant confidentiality. All records that contain names or other personal identifiers, such as locator forms and informed consent forms, will be stored separately from study records identified by code number. All local databases will be secured with password-protected access systems.

\section{Patient and public involvement}

There was no patient or public involvement in the study design.

\section{DISCUSSION}

Hypertension is the most frequent reason for consultation in primary healthcare, representing nearly $6 \%$ of all visits to Spanish family physicians. ${ }^{22}$ The diagnosis of hypertension depends on BP measurements. However, these vary widely, and include several hidden effects, including the white coat effect. Two of the most important international guidelines suggest using 24-hour ABPM. ${ }^{46}$ Despite the validity and reliability of this method, it has drawbacks: a high cost, the lack of instruments to carry out the procedure in all patients, the discomfort of the patient during sleep (the cuff activates every $30 \mathrm{~min}$ at night) and the reluctance of some patients to wear the device for 24 hours. $^{8}{ }^{23}$ Therefore, in daily clinical practice, nurses and physicians use OBPM and HBPM which, while more feasible, lead to other problems such as overdiagnosis. 1-BPM seems to have a sensitivity and specificity in the diagnosis of hypertension close to that obtained with daytime 24-hour ABPM and avoids the white coat effect and other BP effects. Another important aspect that will be examined in this study is the patient experience. In daily practice, clinicians use the best method in any tests requiring medical devices, despite the sensitivity and specificity values, as any diagnostic method requires examination of the patient experience to evaluate the global evidence. ${ }^{24}$

\section{Limitations of the study}

The validation of 1-BPM will not allow night-time recording of $\mathrm{BP}$, a relevant issue in the assessment of cardiovascular risk. Nor does it seek to substitute 24-hour ABPM. However, if the working hypothesis is verified, the new method will allow for a new valid, effective 
diagnostic alternative, acceptable to the patient, and easily applicable in primary healthcare, and more reliable than HBPM and OBPM.

\section{Author affiliations}

${ }^{1}$ Les Corts Primary Health-Care Center, Consorci d'Atenció Primària de Salut

Barcelona Esquerra, Barcelona, Spain

2Primary Healthcare Transversal Research Group, Institut d'Investigacions

Biomèdiques August Pi i Sunyer (IDIBAPS), Barcelona, Spain

${ }^{3}$ Department of Statistics and Operational Research, Universitat Politècnica de Catalunya, Barcelona, Spain

${ }^{4}$ Compte Borrell Primary Health-Care Center, Consorci d'Atenció Primària de Salut Barcelona Esquerra, Barcelona, Spain

${ }^{5}$ Casanova Primary Health-Care Center, Consorci d'Atenció Primària de Salut Barcelona Esquerra, Barcelona, Spain

${ }^{6}$ Hypertension Unit, Servicio de Medicina Interna, Institut Clínic de Medicina Interna i Dermatologia (ICMID), Hospital Clínic de Barcelona, Barcelona, Spain

${ }^{7}$ Pharmacy Service, Hospital Clínic Barcelona, Barcelona, Spain

${ }^{8}$ Management office, Consorci d'Atenció Primària de Salut Barcelona Esquerra,

Barcelona, Spain

\section{Acknowledgements We thank all patients participating in the study.}

Collaborators The 0MBP group are: Monserrat Serrato, Ingrid Vidiella and Elisa Pérez (Les Corts Primary Health-Care Center); Eva Sanchez, María Ortega and Silvia Porcar (Compte Borrell Primary Health-Care Center); Xavier Freixa, Maica Herranz, Berta de Andrés and Elena Lagarda (Casanova Primary Health-Care Center).

Contributors LGP, BK and ASA are all involved in the conception and design of the study. LGP drafted the manuscript. LGP, BK and ASA revised the manuscript for important intellectual content and with MCAB, CC, NG, SR, EB, CSB, JMSM, JBA and ES read and approved the final manuscript.

Funding The project received a research grant from the Carlos III Institute of Health, Ministry of Economy and Competitiveness (Spain), awarded on the 2016 call under the Health Strategy Action 2013-2016, within the National Research Program oriented to Societal Challenges, within the Technical, Scientific and Innovation Research National Plan 2013-2016, with reference Pl16/00660, co-funded with European Union ERDF funds (European Regional Development Fund). The funding source will play no role in the study design, data collection, analysis or interpretation, or the writing of the manuscript. The researchers retain complete independence in the conduct of the study. The evaluation committee will annually inspect study progress and adherence to the study protocol. Information can be viewed at at https://bit.ly/2Q62fB. Study coordinators will report the results annually and the sponsor may audit the study completion and best practices.

Competing interests None declared.

Ethics approval Ethical approval was obtained from the Research Ethics Committee of the Hospital Clínic of Barcelona (Ref. number HCB/2014/0615). Written informed consent will be obtained from eligible participants before any assessment or intervention.

Provenance and peer review Not commissioned; externally peer reviewed.

Open access This is an open access article distributed in accordance with the Creative Commons Attribution Non Commercial (CC BY-NC 4.0) license, which permits others to distribute, remix, adapt, build upon this work non-commercially, and license their derivative works on different terms, provided the original work is properly cited, appropriate credit is given, any changes made indicated, and the use is non-commercial. See: http://creativecommons.org/licenses/by-nc/4.0/.

\section{REFERENCES}

1. Banegas JR, Graciani A, de la Cruz-Troca JJ, et al. Achievement of cardiometabolic goals in aware hypertensive patients in Spain: a nationwide population-based study. Hypertension 2012;60:898-905.
2. Yang Q, Cogswell ME, Flanders WD, et al. Trends in cardiovascular health metrics and associations with all-cause and CVD mortality among US adults. JAMA 2012;307:1273-83.

3. Mills KT, Bundy JD, Kelly TN, et al. Global Disparities of Hypertension Prevalence and Control: A Systematic Analysis of Population-Based Studies From 90 Countries. Circulation 2016;134:441-50.

4. Williams B, Mancia G, Spiering W, et al. ESC/ESH Guidelines for the management of arterial hypertension. Eur Heart $J$ 2018;2018:3021-104.

5. Krause T, Lovibond K, Caulfield M, et al. Management of hypertension: summary of NICE guidance. BMJ 2011;343:d4891

6. Siu AL. U.S. Preventive Services Task Force. Screening for high blood pressure in adults: U.S. Preventive Services Task Force recommendation statement. Ann Intern Med 2015;163:778-86.

7. Piper MA, Evans CV, Burda BU, et al. Diagnostic and predictive accuracy of blood pressure screening methods with consideration of rescreening intervals: a systematic review for the U.S. Preventive Services Task Force. Ann Intern Med 2015;162:192-204.

8. Turner JR, Viera AJ, Shimbo D. Ambulatory blood pressure monitoring in clinical practice: a review. Am J Med 2015;128:14-20.

9. Stergiou GS, Parati G, Vlachopoulos C, et al. Methodology and technology for peripheral and central blood pressure and blood pressure variability measurement. J Hypertens 2016;34:1665-77.

10. Mutlu S, Sari O, Arslan E, et al. Comparison of ambulatory blood pressure measurement with home, office and pharmacy measurements: is arterial blood pressure measured at pharmacy reliable? J Eval Clin Pract 2016;22:40-5.

11. Banegas JR, Ruilope LM, de la Sierra A, et al. Relationship between Clinic and Ambulatory Blood-Pressure Measurements and Mortality. N Engl J Med 2018;378:1509-20.

12. Banegas JR, Segura J, Sobrino J, et al. Effectiveness of blood pressure control outside the medical setting. Hypertension 2007;49:62-8.

13. Little P, Barnett J, Barnsley L, et al. Comparison of agreement between different measures of blood pressure in primary care and daytime ambulatory blood pressure. BMJ 2002;325:254.

14. Gaborieau V, Delarche N, Gosse P. Ambulatory blood pressure monitoring versus self-measurement of blood pressure at home: correlation with target organ damage. J Hypertens 2008;26:1919-27.

15. Viera AJ, Hinderliter AL, Kshirsagar AV, et al. Reproducibility of masked hypertension in adults with untreated borderline office blood pressure: comparison of ambulatory and home monitoring. Am J Hypertens 2010;23:1190-7.

16. Gorostidi M, Banegas JR, de la Sierra A, et al. Ambulatory blood pressure monitoring in daily clinical practice - the Spanish ABPM Registry experience. Eur J Clin Invest 2016;46:92-8.

17. van der Wel MC, Buunk IE, van Weel C, et al. A novel approach to office blood pressure measurement: 30-minute office blood pressure vs daytime ambulatory blood pressure. Ann Fam Med 2011;9:128-35

18. Mas-Heredia M, Molés-Moliner E, González-de Paz L, et al. Validity and applicability of a new recording method for hypertension. Rev Esp Cardiol 2014;67:717-23.

19. Millaret M, Pons JMV. Participació de ciutadans i pacients en les polítiques de salut: conceptualització i experiències internacionals. 1st ed. Generalitat de Catalunya. 2015. available in http://aquas. gencat.cat/web/.content/minisite/aquas/publicacions/2015/ documents/Participacio Ciutadans Pacients_Informe_AQuAS 2015 pdf (accessed 28 Mar 2019).

20. Department of Health \& Human Services. Premarket notification of intent to market the device Oscar 2. https://www.accessdata.fda. gov/cdrh_docs/pdf15/K151520.pdf.

21. Oscar 2 - SunTech Medical. https://www.suntechmed.com/bpproducts/ambulatory-blood-pressure-monitoring/oscar-2.

22. Sicras-Mainar A, Navarro-Artieda R. [Cost of arterial hypertension according to levels of morbidity in primary care setting]. Med Clin 2009:133:290-5.

23. Chrubasik S, Droste C, Glimm E, et al. Comparison of different methods of blood pressure measurements. Blood Press Monit 2007;12:157-66.

24. World Health Organization. Regional Office for Europe. Exploring patient participation in reducing health-care related safety risks. Copenhagen, Denmark: WHO Regional Office for Europe. 2013. Available in http://www.euro.who.int/_data/assets/pdf_file/0010/ 185779/e96814.pdf (accessed 28 Mar 2019). 